\title{
PROCESSUS DIGESTIFS DES RUMINANTS
}

\section{II. - INFLUENCE DES ANTIBIOTIQUES \\ SUR LA DIGESTIBILITE IN VITRO DU FOIN DE LUZERNE}

PAR

\author{
A. C. FRANÇOIS, A. M. LEROY. S. Z. ZELTER (1) \\ Laboratoire de Recherches de Zootechnie, Institut National Agronomique \\ Paris
}

\section{PLAN DU MEMOIRE}

\section{Introduction.}

\section{I. - Méthodes expérimentales.}

Io Technique de digestion.

$2^{0}$ Antibiotiques étudiés.

\section{II. - Résultats.}

a) Influence de doses variables d'auréomycine.

b) Influence de doses croissantes de pénicilline.

c) Influence comparée de divers antibiotiques.

\section{III. - Interprétation et discussion.}

\section{IV. - Résumé et Conclusions.}

Bibliographie.

L'utilisation des antibiotiques dans l'alimentation des volailles, des porcins et des jeunes ruminants a pris, ces dernières années, une grande extension. Un grand nombre de travaux signalent - outre l'effet stimulant sur la croissance - une action sur la microflore du tube digestif des animaux. On peut dès lors se demander quelles sont les conséquences d'une ingestion d'antibiotiques sur les phénomènes digestifs des espèces animales chez lesquelles l'activité bactérienne intervient pour une très large part dans la dégradation du bol alimentaire. Ce problème se pose, en particulier, pour le ruminant. En effet, un travail récent de BELL et collaborateurs (I) signale que 1'administration de $54 \mathrm{mg}$ d'auréomycine par $\mathrm{kg}$ de matière sèche absorbée produit chez un bovin une diminution sensible de la digestibilité de la matière sèche, de la cellulose et des extrac- 
tifs non azotés. Le triple de cette dose provoque des troubles digestifs sérieux. De même, CoLBy et collaborateurs (2) constatent chez le mouton une inappétence prononcée à la suite d'une ingestion de $200 \mathrm{mg}$ d'auréomycine par jour.

La technique d'étude in vitro de la digestion au niveau du rumen que nous avons décrite dans un précédent mémoire (3) nous a permis d'examiner de près les effets de l'adjonction de différents antibiotiques à un même substrat qui, en l'occurrence, était un foin de luzerne, aliment utilisé couramment dans le régime des ruminants.

\section{I. - METHODE EXPERIMENTALE}

\section{$I^{o}$ Technique de digestion}

Les détails expérimentaux et les techniques analytiques ont été décrits ailleurs (3). Précisons, toutefois, que la quantité de substrat utilisé au cours de la présente étude est de $29 \mathrm{~g}$ de foin de luzerne contenant 26,4 $\mathrm{g}$ de matière sèche, $6,92 \mathrm{~g}$ de cellulose, dosée selon la méthode KursCHNER, et $5,36 \mathrm{~g}$ de glucides réducteurs (exprimés en glucose) après hydrolyse à l'ébullition.

Dans chaque essai, 1'effet des antibiotiques introduits a été comparé, toutes conditions identiques par ailleurs, à un témoin constitué de substrat sans antibiotique.

Pour nous assurer de la validité d'une telle comparaison, nous avons vérifié la reproductibilité des résultats en répétant l'ensemencement de plusieurs échantillons du même substrat avec un même inoculum, et en étudiant les modifications subies par le milieu après $4^{8} \mathrm{~h}$ d'incubation à $39^{\circ}$.

Voici les résultats de cette expérience :

TABLEAU I

\begin{tabular}{|c|c|c|c|c|c|c|c|c|}
\hline & \multicolumn{3}{|c|}{ Substances disparues } & \multirow{2}{*}{ 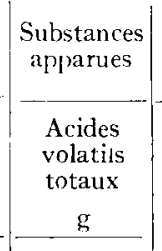 } & \multicolumn{3}{|c|}{$\begin{array}{c}\text { Répartition } \\
\text { des acides volatils } \\
\% \\
\end{array}$} & \multirow[b]{2}{*}{$\mathrm{l}^{\prime H}$} \\
\hline & $\begin{array}{c}\text { Cellulose } \\
\% \\
\end{array}$ & 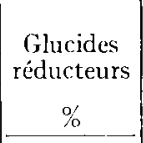 & $\begin{array}{c}\text { Glucides } \\
\text { totaux } \\
\mathrm{g} \\
\end{array}$ & & $\begin{array}{l}\text { Acide } \\
\text { Acé- } \\
\text { tique }\end{array}$ & $\begin{array}{l}\text { Acide } \\
\text { Buty- } \\
\text { rique }\end{array}$ & $\begin{array}{c}\text { Acide } \\
\text { Propio- } \\
\text { nique }\end{array}$ & \\
\hline $\begin{array}{l}\text { Fiole } A \ldots \ldots \\
\text { Fiole } \mathrm{B} \ldots \ldots \ldots \\
\text { Fiole } \mathrm{C} \ldots \ldots \ldots \\
\text { Moyenne } \ldots \ldots . \\
\text { Foart maximum } \\
\text { par rapport à la } \\
\text { moyenne } \ldots . . \\
\text { Ecart entre les } \\
\text { valeurs extrêmes }\end{array}$ & $\begin{array}{c}3^{8,5} \\
40,4 \\
42, \mathrm{I} \\
40,3=\mathrm{I}, 3 \\
4,5 \% \\
9,4 \%\end{array}$ & $\begin{array}{c}69,7 \\
69,7 \\
7 \mathrm{I}, \mathrm{I} \\
70, \mathrm{I}=0,6 \\
1,4 \% \\
2 \%\end{array}$ & $\begin{array}{c}5,06 \\
5, \mathrm{I} 3 \\
5,22 \\
5,14: 0,06\end{array}$ & 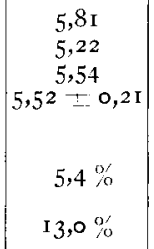 & $\begin{array}{l}57,0 \\
57,4 \\
54,6 \\
56,3\end{array}$ & $\begin{array}{l}35,2 \\
34,2 \\
33,5 \\
34,3\end{array}$ & $\begin{array}{r}7,8 \\
8,5 \\
11,9 \\
9,4\end{array}$ & $\begin{array}{l}5,80 \\
5,70 \\
5,80 \\
5,77\end{array}$ \\
\hline
\end{tabular}


La très faible valeur des erreurs-types des moyennes prouve la fidélité satisfaisante de la technique et justifie la méthode de comparaison que nous avons adoptée pour l'interprétation de nos observations. Toutefois, pour éviter toute interprétation douteuse, nous nous sommes volontairement placés dans les conditions les moins favorables en admettant que les différences entre échantillons de contrôle et d'expérience ne doivent être considérées comme significatives que lorsqu'elles sont au moins égales à Io \% en valeur relative, en ce qui concerne la cellulose. Pour les glucides la différence devient significative 1orsqu'elle est supérieure à $2 \%$, pour les glucides totaux : $3 \%$ et pour les acides volatils I3 $\%$ au moins.

\section{$2^{\circ}$ Antibiotiques étudiés ( $\left.{ }^{1}\right)$}

Les antibiotiques suivants ont été utilisés au cours de ces recherches:

Essais I $a$ et I $b$ : Auréomycine S. A. F.

Essai 2 : Pénicilline (sel de potassium) S. A. F. - Auréomycine (chlorhydrate) R. P. Lot no 376 . - Chloramphénicol R. P. Lot G. P. 672. - Pénicilline G (sel de sodium) R. P. Lot $\mathrm{n}^{\circ} 2808$ D. F. V.

Essai $3 b$ : Auréomycine (chlorhydrate) R. P. Lot no 376 . - Pénicilline $\mathrm{G}$ (sel de Na) R. P. I ot no 2808 D. F. V. - Pénicilline G (sel de procaïne) R. P. I ot no 529 D. F. V.

Essai $3 c$ : Pénicilline G (sel de procaïne) R. P. Lot $n^{\circ} 529$ D. F. V. Dihydrostreptomycine (sulfate) R. P. Lot no 898. Specia (didromycine). - Streptomycine (sulfate) $\mathrm{I}_{1}$ ot $\mathrm{n}^{\mathrm{O}}{ }_{5 \mathrm{I} 2}$ Specia. - Bacitracine S. A. F.

A l'exception de l'auréomycine et du sel de potassium de la pénicilline (essais I $a, \mathrm{I} b$ et 2 ) ainsi que de la pénicilline procaïne, qui ont été étudiés à dose variable, tous les autres antibiotiques ont été ajoutés au substrat à raison de $2 \mathrm{mg}$ par essai, soit $76 \mathrm{mg}$ par $\mathrm{kg}$ de matière sèche de foin de luzerne. Cette dose est d'au moins trois fois supérieure à celle incorporée dans les aliments destinés aux porcins et aux volailles.

L'introduction des antibiotiques dans le milieu d'incubation a été effectuée à l'état de solution aqueuse préparée au moment de l'ensemencement du milieu.

\section{II. - RÉSULTATS}

\section{a) Influence de doses variables d'auréomycine (essais I $a$ et $\mathrm{x} b$ )}

Les doses introduites varient de $0,25 \mathrm{mg}$ à $2 \mathrm{mg}$ par essai, soit de 9,5 à $76 \mathrm{mg}$ par kg de matière sèche.

(1) Nous remercions la Société des Antibiotiques de France et la Société Rhône-Poulenc d'avoir bien voulu nous procurer les antibiotiques nécessaires ì nos recherches. 
Le tableau II rapporte les résultats.

\section{TABLEAU II}

Influence de doses croissantes d'auréomycine

\begin{tabular}{|c|c|c|c|c|c|c|c|c|c|c|}
\hline \multirow[b]{2}{*}{ Essai } & \multirow[b]{2}{*}{$\begin{array}{l}\text { Dose } \\
\text { mg }\end{array}$} & \multicolumn{3}{|c|}{ Substances disparues } & \multicolumn{2}{|c|}{$\begin{array}{l}\text { Substances } \\
\text { apparues }\end{array}$} & \multicolumn{3}{|c|}{$\begin{array}{c}\text { Répartition des acides } \\
\text { volatils } \%\end{array}$} & \multirow[b]{2}{*}{$\begin{array}{l}\mathrm{pH} \\
\text { (9) }\end{array}$} \\
\hline & & $\begin{array}{c}\text { Cellu- } \\
\text { lose } \\
\% \\
(\mathrm{I})\end{array}$ & $\begin{array}{c}\text { Gluci- } \\
\text { des ré-- } \\
\text { ducteurs } \\
\% \\
(2)\end{array}$ & $\begin{array}{c}\text { Gluci- } \\
\text { des } \\
\text { totaux } \\
\mathrm{g} \\
(3)\end{array}$ & $\mid \begin{array}{c}\text { Acide } \\
\text { lactique } \\
g \\
(4)\end{array}$ & $\begin{array}{c}\text { Acides } \\
\text { volatils } \\
g \\
(5)\end{array}$ & $\begin{array}{l}\text { Acéti-- } \\
\text { que } \\
(6)\end{array}$ & $\begin{array}{c}\text { Propio- } \\
\text { nique } \\
(7)\end{array}$ & $\begin{array}{l}\text { Buty- } \\
\text { rique } \\
(8)\end{array}$ & \\
\hline I $a$ & $\begin{array}{l}\circ \\
0,25(1) \\
\text { I,00 }\end{array}$ & $\begin{array}{l}45,1 \\
39,8 \\
I I, 7\end{array}$ & $\begin{array}{l}70,9 \\
70,8 \\
61,1\end{array}$ & $\begin{array}{l}6,921 \\
6,523 \\
4,094\end{array}$ & $\stackrel{0,01}{-}$ & $\begin{array}{l}5,75 \\
5,57 \\
5,07\end{array}$ & $\begin{array}{l}63,5 \\
56,6 \\
65,5\end{array}$ & $\begin{array}{l}24,6 \\
32,5 \\
25,7\end{array}$ & $\begin{array}{r}\text { I I,9 } \\
10,9 \\
9,8\end{array}$ & $\begin{array}{l}5,75 \\
5,85 \\
6,10\end{array}$ \\
\hline I $b$ & $\begin{array}{l}\circ \\
1,00 \\
2,00\end{array}$ & $\begin{array}{l}33,6 \\
23,5 \\
23,5\end{array}$ & $\begin{array}{l}73,3 \\
71,7 \\
60,1\end{array}$ & $\begin{array}{l}6,256 \\
5,473 \\
4,851\end{array}$ & - & $\begin{array}{l}3,37 \\
4,03 \\
3,17\end{array}$ & $\begin{array}{l}60,3 \\
59,9 \\
74,1\end{array}$ & $\begin{array}{l}33,2 \\
40,1 \\
25,9\end{array}$ & $\stackrel{6,5}{-}$ & $\begin{array}{l}5,65 \\
6,05 \\
5,90\end{array}$ \\
\hline
\end{tabular}

(1) Moyenne de deux essais.

\section{b) Influence de doses croissantes de pénicilline (sel de potassium)}

(Essai 2)

Au cours de cet essai, dont les résultats figurent dans le tableau III, les doses d'antibiotique introduites varient également dans cette expérience de $0,25 \mathrm{mg}$ à $2,00 \mathrm{mg}$.

TABI,EAU III

Influence de doses croissantes de pénicilline

\begin{tabular}{|c|c|c|c|c|c|c|c|c|c|c|}
\hline \multirow[b]{2}{*}{ Essai } & \multirow[b]{2}{*}{$\begin{array}{l}\text { Dose } \\
\text { mg }\end{array}$} & \multicolumn{3}{|c|}{ Substances disparues } & \multicolumn{2}{|c|}{$\begin{array}{c}\text { Substances } \\
\text { apparues }\end{array}$} & \multicolumn{3}{|c|}{$\begin{array}{l}\text { Répartition des acides } \\
\text { volatils } \%\end{array}$} & \multirow[b]{2}{*}{$\mathrm{pH}$} \\
\hline & & $\begin{array}{c}\text { Cellu- } \\
\text { lose } \\
\% \\
\text { (1) }\end{array}$ & $\begin{array}{c}\text { Gluci- } \\
\text { des ré- } \\
\text { ducteurs } \\
\% \\
\% \\
(2) \\
\end{array}$ & $\begin{array}{c}\text { Gluci- } \\
\text { des } \\
\text { totaux } \\
g \\
(3) \\
\end{array}$ & $\begin{array}{c}\text { Acide } \\
\text { lactique } \\
\mathrm{g} \\
(4)\end{array}$ & \begin{tabular}{|c} 
Acides \\
volatils \\
$\mathrm{g}$ \\
$(5)$ \\
\end{tabular} & $\begin{array}{c}\text { Acéti- } \\
\text { que } \\
(6)\end{array}$ & $\begin{array}{c}\text { Propio- } \\
\text { nique } \\
(7)\end{array}$ & $\begin{array}{c}\text { Buty- } \\
\text { rique } \\
\text { (8) } \\
\end{array}$ & \\
\hline 2 & $\begin{array}{l}\circ \\
0,25 \\
0,50 \\
1,00 \\
2,00\end{array}$ & $\begin{array}{l}42,8 \\
44,8 \\
40,7 \\
42,0 \\
45,0\end{array}$ & $\begin{array}{l}58,0 \\
60,5 \\
60,9 \\
60,7 \\
58,6\end{array}$ & $\begin{array}{l}6,070 \\
6,357 \\
6,095 \\
6,166 \\
6,259\end{array}$ & $\begin{array}{l}\bar{z} \\
\overline{-} \\
\overline{-}\end{array}$ & $\begin{array}{l}6,36 \\
6,41 \\
6,14 \\
6,34 \\
6,22\end{array}$ & $\begin{array}{l}66,7 \\
68,0 \\
59,6 \\
63,5 \\
60,3\end{array}$ & $\begin{array}{l}27,2 \\
27,9 \\
32, \mathrm{I} \\
29,4 \\
3 \mathrm{I}, 8\end{array}$ & $\begin{array}{l}6, \mathbf{I} \\
4, \mathbf{I} \\
8,3 \\
7, \mathbf{I} \\
7,9\end{array}$ & $\begin{array}{l}5,55 \\
5,55 \\
5,65 \\
5,80 \\
5,65\end{array}$ \\
\hline
\end{tabular}

c) Influence comparée des divers antibiotiques : auréomycine, chloramphénicol, sel de sodium de la pénicilline, sel de procaine de la pénicilline, dihydrostreptomycine, streptomycine, bacitracine (essais $3 a$, $3 b, 3 c)$. 
Tous ces antibiotiques ont été utilisés à la dose de $2 \mathrm{mg}$ par essai. Cependant, dans le cas de la pénicilline procaïne, nous avons fait également un essai avec $4 \mathrm{mg}$ (essai $3 b$ ) pour tenir compte du fait que la quantité d'antibiotique présente dans ce sel est environ la moitié de celle qui figure dans les sels de sodium et de potassium du même antibiotique.

Les résultats sont consignés dans le tableau IV.

\section{'TABLEAU IV}

\section{Influence comparée d'antibiotiques divers}

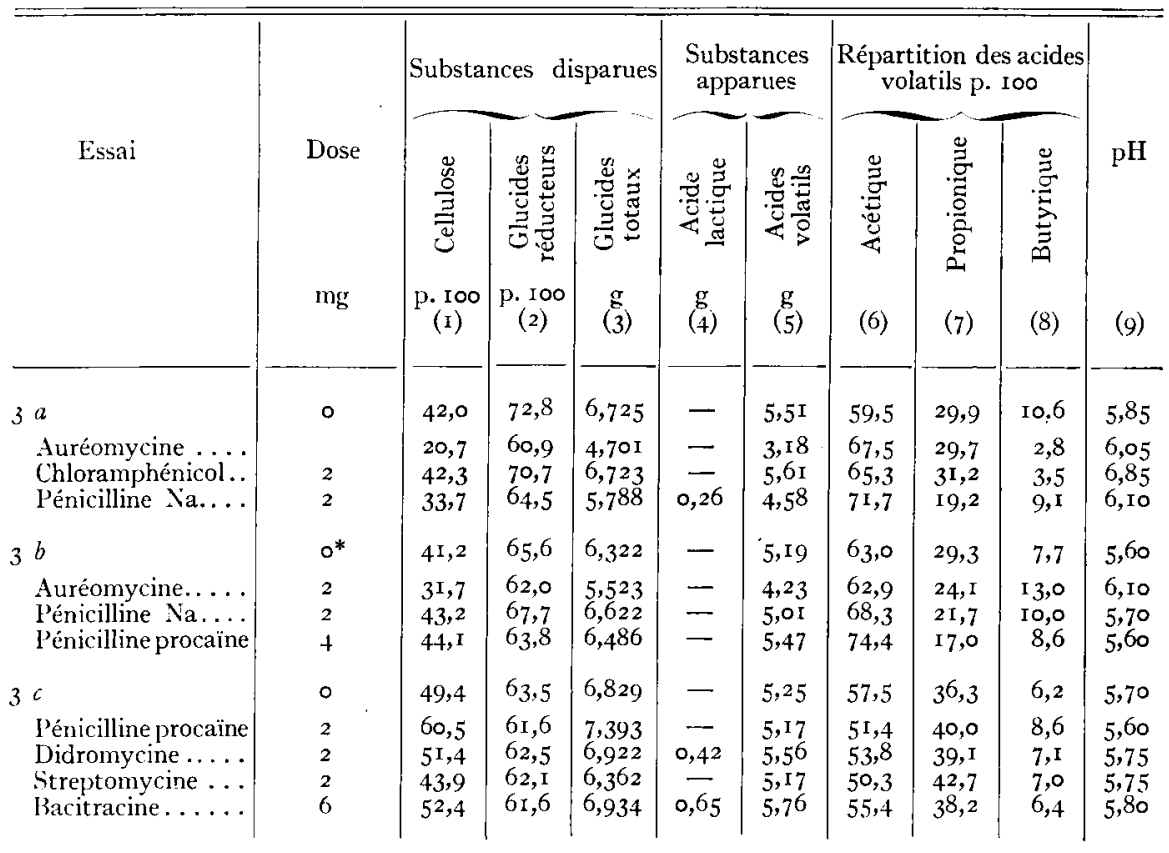

* Moyenne de deux essais.

\section{III. - INTERPRETATION ET DISCUSSION}

Afin de dégager avec netteté l'influence individuelle des différents antibiotiques sur la digestion in vitro $\mathrm{du}$ foin de luzerne, nous poserons que les quantités de chacune des substances disparues ou apparues, dosées dans le témoin à la fin de l'incubation, sont égales à roo, et nous exprimerons tous les autres résultats rapportés à cette base, en p. Ioo (voir tableau V). 


\section{Tableau V}

Comparaison de l'action des différents antibiotiques sur la digestion in vitro $d u$ foin de luzerne (le témoin est pris égal à 100)

\begin{tabular}{|c|c|c|c|c|c|c|}
\hline \multirow[b]{2}{*}{ Essai } & & \multicolumn{3}{|c|}{ Disparus } & \multicolumn{2}{|c|}{ Apparus } \\
\hline & & Cellulose & $\begin{array}{l}\text { Glucides } \\
\text { hydroly- } \\
\text { sables }\end{array}$ & $\begin{array}{l}\text { Glucides } \\
\text { totaux }\end{array}$ & $\begin{array}{l}\text { Acides } \\
\text { volatils } \\
\text { totaux }\end{array}$ & $\begin{array}{l}\text { Acide } \\
\text { lactique }\end{array}$ \\
\hline $\begin{array}{c}\text { témoin } \\
\text { Auréonycine ........... }\end{array}$ & & 100 & I00 & 100 & 100 & Absence \\
\hline I $a \ldots \ldots \ldots \ldots \ldots \ldots \ldots$ & $0,25 \mathrm{mg}$ & 88,0 & 99,9 & 94,2 & 96,8 & - \\
\hline I $a \ldots \ldots \ldots \ldots \ldots \ldots \ldots \ldots$ & $1,00-$ & 25,9 & 86.5 & $59, \mathrm{I}$ & 88,2 & 一 \\
\hline I $b \ldots \ldots \ldots \ldots \ldots \ldots \ldots$ & $1,00-$ & 69,9 & 98,0 & 87,4 & I 19,5 & 一 \\
\hline I $b \ldots \ldots \ldots \ldots \ldots \ldots \ldots$ & $2,00-$ & 69,9 & 82,2 & 77,5 & 94,0 & $\ldots$ \\
\hline 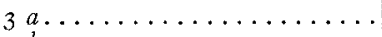 & $2,00-$ & 49,3 & 83,6 & 69,9 & 57,6 & - \\
\hline $\begin{array}{r}3 \text { b.n } \\
\quad \text { Pénicilline (sel de } \mathrm{K}) \ldots \ldots\end{array}$ & $2,00-$ & 77,0 & 94,5 & 87,4 & $8 \mathrm{I}, 5$ & - \\
\hline${ }_{2} \ldots \ldots \ldots \ldots \ldots \ldots$ & $0,25 \mathrm{mg}$ & IO 4,5 & 104,2 & IO 4,4 & 100,8 & - \\
\hline $2, \ldots \ldots \ldots \ldots \ldots \ldots \ldots \ldots$ & $0,50-$ & $95, \mathrm{I}$ & 105,0 & 100,2 & 96,5 & -- \\
\hline $2, \ldots \ldots \ldots \ldots \ldots \ldots \ldots \ldots$ & $1,00-$ & 98,0 & 104,6 & 101,5 & 99,7 & - \\
\hline $\begin{array}{r}\quad \ldots \ldots \ldots \ldots \ldots \\
\quad \text { Pénicilline (sel de } \mathrm{Na}) \ldots \ldots\end{array}$ & $2,00-$ & 105,0 & 101,0 & $103, \mathrm{I}$ & 97,6 & - \\
\hline $\begin{array}{l}3 a \ldots \ldots \ldots \ldots \ldots \ldots \ldots \ldots \ldots \ldots \ldots \ldots \\
3 \\
b \ldots \ldots \ldots \ldots \ldots \ldots \ldots \ldots\end{array}$ & $2,00 \mathrm{mg}$ & $\begin{array}{r}80,2 \\
105,0\end{array}$ & $\begin{array}{r}88,7 \\
103,0\end{array}$ & $\begin{array}{r}86, \text { I } \\
\text { I } 04,7\end{array}$ & $\begin{array}{l}8,0 \\
96,5\end{array}$ & Présence $\left({ }^{\perp}\right)$ \\
\hline Pénicilline (sel de procaïne). . & & & & & & \\
\hline $\begin{array}{l}3 c \ldots \ldots \ldots \ldots \ldots \ldots \ldots \ldots \\
3 \quad b \quad \ldots \ldots \ldots \ldots \ldots \ldots\end{array}$ & $\stackrel{2,00 \mathrm{mg}}{4,00}$ & $\begin{array}{l}\text { I } 22,5 \\
107,0\end{array}$ & $\begin{array}{l}97, \mathbf{I} \\
97,2\end{array}$ & $\begin{array}{l}108,0 \\
102,5\end{array}$ & $\begin{array}{r}98,6 \\
105,7\end{array}$ & - \\
\hline Divers : & & & & & & \\
\hline $3 a$ Chloramphénicol . & $2,00 \mathrm{mg}$ & 100,8 & 97,2 & IOO,O & I02,0 & - \\
\hline $3<$ Didromycine ....... & $2,00-$ & 104,0 & 98,5 & IOI, 2 & 105,9 & Présence $\left({ }^{2}\right)$ \\
\hline $3 c$ Streptomycine $\ldots \ldots \ldots \ldots$ & $2,00-$ & 89,0 & 97,9 & 93,2 & 98,6 & - \\
\hline $3<$ Bacitracine $\ldots \ldots \ldots \ldots$ & $2,00-$ & $106, \mathrm{I}$ & $97, \mathbf{I}$ & IOI,5 & 109,8 & Présence $\left({ }^{3}\right)$ \\
\hline 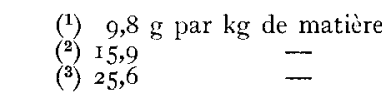 & sèche de & ostrat. & & & & \\
\hline
\end{tabular}

\section{a) Auréomycine}

Cet antibiotique exerce une action dépressive à partir de la dose de $38 \mathrm{mg}$ par $\mathrm{kg}$ de matière sèche de substrat, action qui se manifeste déjà à la dose de 9,5 mg. L,e phénomène est particulièrement significatif pour la cellulose dont le taux de disparition se trouve abaissé en moyenne d'environ $35 \%$, lorsque la dose d'auréomycine ajoutée atteint $76 \mathrm{mg}$ par $\mathrm{kg}$ de matière sèche. La dépression affecte, dans une moindre mesure, les glucides hydrolysables, dont la diminution de digestibilité, à la même dose

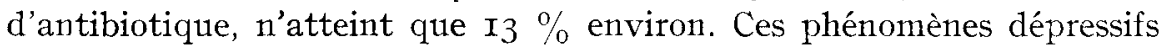


se soldent par une moindre quantité d'acides volatils formés aux dépens des glucides disparus (tableau II, IV, V). Ainsi, tandis que la quantité d'acides volatils totaux formés par kg de matière sèche de foin de luzerne non traité est de $178 \mathrm{~g}$, celle apparue dans ce même substrat additionnée d'auréomycine $(76 \mathrm{mg} / \mathrm{kg})$ est de $\mathrm{I} 34 \mathrm{~g}$ seulement.

Le pH plus élevé observé dans le substrat traité à l'auréomycine corrobore, par ailleurs, cette différence. Cette constatation in vitro, dont les conséquences chez le ruminant sont importantes en raison du rôle physiologique de ces acides, s'accorde parfaitement avec les observations faites in vivo par BEIL et coll. (I). Quoique nos données, relatives aux doses de I mg par essai soient plus discordantes que les autres, elles indiquent, cependant, dans ce cas, également une dépression de digestibilité.

\section{b) Pénicilline}

I es tableaux III et V montrent que, sous forme de sel de potassium, ajouté à la dose de $7^{6} \mathrm{ing}$ par $\mathrm{kg}$ de matière sèche de substrat, cet antibiotique n'exerce pas d'action significative sur la dégradation glucidique ou sur la formation d'acides volatils.

I a divergence des résultats obtenus avec le traitement au sel de soditum de la pénicilline (essais $3 a$ et $3 b$ ) ne permet pas de se prononcer sur l'effet produit par cet antibiotique.

En revanche, à l'état de sel de procaïne, la pénicilline semble stimuler légèrement la cellulolyse. I a production d'acides volatils ne paraît toutefois pas être fortement influencée.

\section{c) Antibiotiques divers (chloramphénicol, dihydrostreptomycine, streptomycine, bacitracine)}

A l'exception de la streptomycine, qui déprimerait légèrement la dégradation de la cellulose, les autres antibiotiques de cette série sont sans action sur la digestibilité in vitro. Signalons, toutefois, que la dihydrostreptomycine et la bacitracine semblent favoriser l'apparition de petites quantités d'acide lactique dans le milieu.

Quoique ayant utilisé des doses d'antibiotiques supérieures aux nôtres, WASSERMAN et coll. (4) concluent comme nous que la cellulolyse dans le rumen n'est pas affectée par la pénicilline et que la streptomycine, en revanche, exerce une action dépressive. Signalons que l'auréonycine n'avait pas été étudiée par ces auteurs.

Nos résultats montrent que l'auréomycine ne semble pas être inactivée par le liquide du rumen. BELr et collaborateurs (I) ont montré que la pénicilline est rapidement inactivée par ce milieu, ainsi que la chloromycé- 
tine. En revanche, le taux de streptomycine semble s'accroitre pendant l'incubation. Ces observations s'accordent bien avec les nôtres, qui montrent que 1a streptomycine exerce une légère influence dépressive sur la digestibilité de la cellulose.

L'évolution du milieu de rumen sous l'action d'une administration répétée d'antibiotique ne semble pas avoir été étudiée jusqu'à présent et fera l'objet de recherches ultérieures.

\section{IV. - RÉSUMÉ ET CONCLUSIONS}

L'influence de quelques antibiotiques (auréomycine, diverses formes de pénicilline, chloramphénicol, dihydrostreptomycine, streptomycine, bacitracine) sur la digestibilité in vitro d'un foin de luzerne a été étudiée. Les résultats montrent que des doses d'auréomycine comprises entre 9.5 et $76 \mathrm{mg}$ par $\mathrm{kg}$ de matière sèche de substrat dépriment sensiblement à la fois la dégradation des glucides (plus particulièrement de la cellulose) et la formation d'acides gras volatils.

La streptomycine exerce également une légère action dépressive sur 1a cellulolyse. A l'exception de la pénicilline-procaïne, qui semble stimuler légèrement la digestibilité, les autres antibiotiques sont sans effet aux doses utilisées $(9,5$ à $76 \mathrm{mg}$ par $\mathrm{kg}$ de matière sèche).

(Reçu pour publication le 5 juin 1953.)

\section{BIBLIOGRAPHIE}

(I) Beil. (M. C.), Whitehair (C. K.), Gallup (W. D.). - The effect of aureomycine on digestion in steers. J. of Animal Science, 9, p. 647-648, I950.

(2) Colby (R. W.), RaU (F. A.), Dunn (R. C.). - The effect of aureomycine on rumen flora of sheep. Proc. Soc. Exptl. Biol. Med., 75, p. 234-236, I950.

(3) Fauconneau (G.), François (A. C.), Leroy (A. M.), Zeiter (S. Z.). Processus digestifs des ruminants. I. - Etude in vitro de la digestion du foin de luzerne. Ann, Zoot., 2 p. 275-284, I953.

(4) Wassermann (R. H.), Duncan (C. W.), Churchill (E. S.), Huffman C. F.). - The effects of antibiotics in-vitro cellulose digestion by rumen, microorganisms. J. of Dairy Sc., 35, no 7, p. 57I-579, 1952. 